\title{
BLISTER FARMACÊUTICO: A INFLUÊNCIA DA COR E DOS TIPOS DE FILMES NO ACONDICIONAMENTO DE MEDICAMENTOS
}

\section{PHARMACEUTICAL BLISTER: INFLUENCE OF COLOR AND TYPES OF FILMS IN DRUG PACKAGING}

\section{Diênifer Alves Caixeta PEREIRA ${ }^{1}$; Lilian Abreu FERREIRA²}

\author{
1 - Acadêmica do curso de Farmácia, Faculdade Patos de Minas \\ 2 - Docente Mestre Faculade de Farmácia, Faculdade Patos de Minas, Minas Gerais \\ Autor para correspondência: dienifer_caixeta@outlook.com.br
}

\section{RESUMO}

É fundamental a seleção adequada do blister no acondicionamento de um medicamento, de forma a garantir a sua integridade e proteção aos fatores externos. Assim, o objetivo do estudo foi realizar uma pesquisa bibliográfica de caráter exploratório acerca do blister como embalagem farmacêutica e os tipos de materiais e cores a ele relacionados, através de uma busca nas bases Pudmed, SciELO e Google Acadêmico. Com o avanço industrial, o blister tem ocupado um maior segmento entre os tipos de embalagens farmacêuticas. Alguns materiais como o PVC, são bastante utilizados na fabricação do blister, possuindo baixo custo e boa termoformabilidade, entretanto não correspondem às expectativas quanto se faz necessário uma barreira maior a umidade para fármacos altamente higroscópicos. Nestes casos,é recomendado o uso de materiais com uma função de proteção maior, como os laminados combinados PVC/PVDC, PVC/COC/PVC eAclar ${ }^{\circledR}$. Filmes do tipo Alumino/Alumínio oferecem resultados mais satisfatórios quando se faz necessária uma alta proteção contra a luz, umidade e oxigênio, porém o alto custo limitam seu uso à fármacos altamente sensíveis. A coloração do blister também é uma alternativa muito utilizada para fármacos fotossensíveis, como a nifedipina, corticosteróides, antibióticos, e algumas preparações contendo vitaminas, podendo oferecer, dependendo da cor escolhida, uma redução de 30 a $40 \%$ no grau de fotodegradação. Diante disso, a seleção do blister farmacêutico para qualquer que seja a sensibilidade do fármaco, é fator primordial, para que a embalagem cumpra sua função precípua que é de proteger o medicamento permitindo que este chegue de forma íntegra ao consumidor.

Palavras-chave: Estabilidadede Medicamentos. Embalagem de Medicamentos.

Permeabilidade.

\section{ABSTRACT}

The appropriate selection of the blister in the development of a drug, its fundamental to ensure its integrity and protection to external factors. Thus, the objective of the study was to conduct a literature review about blister as pharmaceutical packaging in the light of its types of materials and colors related to it through a seach on Pubmed, SciELO and Google Schoolar. Some materials such as PVC, have low cost and good thermo formability, however, do not match the expectations of a higher barrier is necessary against moisture for highly hygroscopic drugs. In these cases, it is recommended to use materials with a greater barrier function as laminates combined PVC/PVDC, PVC/COC/PVC and Aclar ${ }^{\circledR}$. Materials like Aluminium/Aluminium offer more satisfactory results when a high protection 
against light, moisture and oxygen is required, but the high cost of film, equipment and process difficulty, limit its use to highly sensitive drugs. Thecolor of the blister is also an alternative widely used for photosensitive drugs such as nifedipine, corticosteroids, antibiotics such as tetracycline and some preparations containing vitamins, which can provide, depending on the chosen color, a reduction of 30 to $40 \%$ in photobleaching degree. Therefore, the selection of pharmaceutical blister whatever the sensitivity of the drug, it is crucial factor for the package to fulfill its primary function that is to protect the product allowing it reaches full so the consumer.

Keywords: Drug Estability. Drug Packaging. Permeability.

\section{INTRODUÇÃO}

A escolha da embalagem adequada está intrinsecamente ligada a estabilidade do medicamento. Esta decisão deve ser parte da pesquisae desenvolvimento dos medicamentos considerando as suas características específicas. O medicamento define as condições específicas de armazenamento e, portanto, a embalagem deve fornecer uma barreira aos fatores externos, a fim de prolongar o prazo de validade deste. Além disso, a embalagem deve ser constituída de materiais que não irão interagir com o produto farmacêutico (STINGHEL, 2014; JORGE, 2013; QUINTAS, 2013; AMARAL, RODRIGUES \&CASTRO, 2012; RODRIGUES \& FERRAZ, 2007; BRASIL, 2005).

Até o século XIX, os medicamentos eram armazenados em vidros, aglomerados em algodão. Em 1963, a InterpackHassia lançou o primeiro blister para a pílula anticoncepcional da Schering (GARCIA, 2001). A razão mais importante paraa introdução do blister no mercado farmacêutico era oferecer aos pacientes uma dose unitária facilmente identificável, permitindo ao paciente verificar se tinha tomado o medicamento prescrito em um determinado dia.Mais do que isso, os medicamentos não administrados permaneciam protegidos contra as condições adversas externas, podendo armazená-los de forma mais conveniente. Não muito longe disso, a indústria farmacêutica foi reconhecendo outras vantagens do blister, tais como redução de custos, redução de acidentes com embalagens de vidro e consequentes quebras, maior comprovação de uso indevido com o blister em comparação à embalagem convencional e facilidade de identificação de violação(PILCHIK, 2000).

Todas essas vantagens explicam o motivo pelo qual somente na Europa, $85 \%$ dos medicamentos sólidos são acondicionados em blister. Nos Estados Unidos, a utilização do blister como forma de embalagem farmacêutica manteve um crescimento constante desde seu início. No Brasil, o blisterocupa o maior segmento, seguido por frascos de plástico soprado e ampolas (AMARAL, RODRIGUES \& CASTRO, 2012; NASCIMENTO, 2006; BERASAY, 2005; PILCHIK, 2000). 
Assim, o objetivo foi realizar uma pesquisa bibliográfica de caráter exploratório acerca da embalagem farmacêutica do tipo blister e os tipos de materiais e cores a ele relacionados.

\section{METODOLOGIA}

Trata-se de um estudo bibliográfico narrativo e exploratório. Foi realizada uma busca de artigos nas bases, Pubmed, SciELO e Google acadêmico, nas línguas portuguesa e inglesa, utilizando as palavras-chaves, embalagem de medicamentos, blister farmacêutico e filme termoformado.

\section{BLISTER FARMACÊUTICO}

A palavra blister tem origem do inglês e significa bolha, sendo formado por uma cartela base (papel, alumínio ou plástico), onde as cápsulas ou comprimidos são colocados nos alvéolos e seladas por um filme alumínio.No Brasil, são encontrados blistersacondicionando especialmente comprimidos, drágeas, cápsulas duras e moles nos mais diferenciados formatos (AMARAL, RODRIGUES \& CASTRO, 2012; CARREIRA, 2009; NICOLOSI, 2008; GARCIA, 2001; FORCÍNIO, 2002).

Há dois tipos básicos de embalagem em blister para produtos farmacêuticos, um onde a cavidade é construída em plástico termomoldável e o verso é formado por um plástico ou uma combinação de plástico, papel e/ou alumínio, e outro ondea embalagem emblister contém alumínio em ambos os lados e sua cavidade é formada por alongamento a frio(RODRIGUES \& FERRAZ, 2007; PILCHIK, 2000).

Entre as vantagens do emprego da embalagem em blister estão, a integridade do produto, onde os medicamentos nele contidos estão hermeticamente selados em seu próprio alvéolo e protegidos contra fatores externos, garantindoproteção do medicamento desde sua fabricação até a utilização do consumidor final e aevidência de violação, poisas formas farmacêuticas são seladas individualmente, tornando imediatamente visível qualquer forma de violaçãoe baixa possibilidade de overdose. O pequeno tamanho da embalagem em blister também facilita o transporte contribuindo para a adesão ao tratamento(RODRIGUES \& FERRAZ, 2007).

Outras vantagens do emprego da embalagem em blister são, boa aparência, hermeticidadedo sistema de fechamento epossibilidade de impressão de informações como 
validade, lote, posologia, entre outros, diretamente na folha de selagem do blister, bem como impressão de cartelas associadas a calendários para controle da administração e provisão de doses únicas identificáveis. Além disso, oferece baixos volumes de estoque no âmbito da indústria, facilidade para o paciente no armazenamento, baixo índice de contaminação microbiológica e economia no transporte(QUINTAS, 2013; AMARAL, RODRIGUES \& CASTRO, 2012).

\subsection{Tipos de Filmes}

Há um blister específico para cada tipo de cápsula ou comprimido que deve ser utilizado.Existem diferentes materiais, diferentes espessuras e as mais variadas cores. Assim, deve-se considerar o tipo de resistência que deverá ter o blister levando-se em consideraçãoas características do produto a ser blistado(GARCIA, 2001).

\subsubsection{Cloreto de Polivinila (PVC)}

É um material transparente, de baixo custo, com alta termomoldabilidade, resistência física e química, baixa permeabilidade a óleos, gorduras e substâncias aromáticas e baixo índice de permeabilidade a umidade do ar. O PVC é o material mais utilizado para embalagem em blister, chegando a $80 \%$ dos casos, entretanto,dentre todos os materiais utilizados é o que oferece menor proteção. Dentro dos $20 \%$ restantes, temos o PVC/PVDC, Aclar® e ALU/ALU respectivamente (CRONIMO GROUP, 2015; GARCIA, 2001; RODRIGUES \& FERRAZ, 2007).

A espessura do filme rígido de PVC geralmente utilizada pelas indústrias farmacêuticas é de $0,2 \mathrm{~mm}$ a $0,8 \mathrm{~mm}$.A espessura possui grande influência na propriedade de barreira à luz, pois quanto maior a espessura, menor é a porcentagem de transmissão de luz que atravessa o material (ALVES et al., 2008; RODRIGUES \& FERRAZ, 2007).

Quanto à aplicação, o PVC transparente, que é o tipo de material mais simples, possui barreira limitada, e somente será utilizado em fármacos mais estáveis em presença de umidade ou luz, como por exemplo, o Paracetamol e o Cotrimoxazol, muito embora alguns fabricantes possam colocar aditivos que potencializem a barreira, como absorvedores de luz UV (JNANADEVA, 2016; REMINGTON, 2004; GARCIA, 2001).

Com o uso do PVC há liberação de toxinas durante sua combustão, e este fato tem levado à sua substituição pelo Polipropileno (PP) para embalagens em blister na Europa. 
Polietileno tereftalato (PET) e poliestireno (PS) podem substituir o PVC, entretanto a alta permeabilidade a umidade comparada ao PVC restringe o uso (PHILCHIK, 2000; HOLLANDER, 1998).

O PP possui alto índice de encolhimento durante a termoformagem, o que o torna um dos mais difíceis de moldar. O PPé mais frequentemente utilizado para acondicionamento de supositórios. (GARCIA, 2001). Ademais, ele é inadequado para o acondicionamento de produtos farmacêuticos fotossensíveis, pois possui uma possui transmitância de até $80 \%$ entre 300 e $400 \mathrm{~nm}$, diferindo do requerido atualmente na Farmacopeia, uma transmitância de, no máximo, 25\% e 20\%,respectivamente, numa faixa de comprimento de onda entre 290 e 450 nm (ALVES et al., 2008).

\subsubsection{Cloreto de polivinilideno (PVDC)}

O PVDC é usado em embalagens com laminação ou revestimento sobre o PVCreduzindo de 5 a 10 vezes a permeabilidade do PVC ao oxigênio e à umidade (STINGHEL, 2014; RODRIGUES \& FERRAZ, 2007).O filme moldável geralmente é incolor e transparente, mas para proteger produtos sensíveis à luz pode ser escurecido. O PVDC não pode ser utilizado como único material devido ao seu custo relativamente elevado e às suas propriedades mecânicas. As melhores propriedades são obtidas pela combinação de PVDC com uma camada base de PVC, utilizado para produtos não muito sensíveis aos fatores externos e com bom tempo de vida útil, como os multivitamínicos em cápsulas ou comprimidos(JNANADEVA, 2016; NÓBREGA et al., 2006; PILCHIK, 2000; HUNTE, 1999).

\subsubsection{PCTFE - Policlorotrifluoretileno (ACLAR®)}

Os filmes do tipo PCTFEpodem ser laminadosao filme de PVCproporcionando embalagens com alta barreira à umidade, sendo esta barreiraparecida com aquela fornecida pelo alumínio, apresentando a transparência nas embalagens que também podem ser escurecidas e o tamanho reduzido final do blistercomo vantagens, após processo de moldagem(RODRIGUES \& FERRAZ, 2007;GARCIA, 2001; LIMA, 2010).

\subsubsection{Copolímeros de Olefinas Cíclicas (COC)}

O COC é parte da família das olefinas e oferece excelente transparência, alta rigidez e boa termofomabilidade. Possui elevada barreira contra umidade (cerca de10 vezes 
a mais que o PVC e mais que o dobro do PVDC revestidode PVC), boa resistência química e biocompatibilidade, mas são incompatíveis com hidrocarbonetosalifáticos ou aromáticos e certas gorduras e óleos.

Estão disponíveis no mercado os laminados PVC-COC-PVC e COC-PVDC para medicamentos que necessitemde alta barreira ao oxigênio e umidade. Laminados associados ao Polipropileno (PP) como o PP-COC-PPvem sendo bastante utilizados em substituição ao PVC e PVDC por estes serem prejudiciais aomeio ambiente (NICOLOSI, 2008; NASCIMENTO, 2006 ;LUSINAet al., 2005).

\subsubsection{Poliamida orientada (OPA)/Alumínio/PVC}

Dependendo da densidade do filme OPA/Alumínio/PVCé possível eliminar quasecompletamente a permeabilidade a umidade. Assim como asestruturas contendo alumínio, ele é moldado a frio. Utilizado em fármacos extremamente sensíveis como a Cefuroximae o Dicloridrato de Levocetirizina(RODRIGUES \& FERRAZ, 2007).

\subsubsection{Alumínio/Alumínio (Alu/Alu®, ColdFormFoil - CFF)}

Pode ser encontrado no mercado com $20 \mu$ a $25 \mu$ de espessura, fornecendo barreira total contra a umidade, oxigênio e a luz.Ao contrário dos blisters contendomaterial plástico, estes não são moldados a quente, mas moldados no formato desejado por pressão a frio.O processo é significativamente mais caro que o processo a quente,além dos alvéolos serem maiores comparados a este, aumentandoassim a área total da embalagem e ocusto, poishá um consumo maior para acondicionar a mesma quantidade de produto que um blister termomoldado como demonstrado na Figura 1 (RODRIGUES \& FERRAZ, 2007; PHILCHIK, 2000).

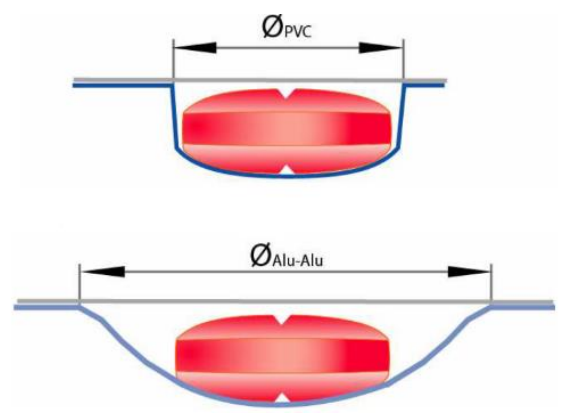

Figura 1 - Diferenças nas cavidades entre o PVC termomoldado e o Aluminío moldado a frio Fonte: CRONIMO GROUP, 2015 


\subsection{A influência da coloração}

A transparência nos filmes pode ser usada como ferramenta de marketing para divulgar as cápsulas ou comprimidos. Também é útil no controle de processo, já que a ausência de comprimidos ou cápsulas pode ser mais facilmente identificada em um blister transparente (Garcia, 2001).

O blíster coloridoé também muito utilizado para fármacos fotossensíveis, tais como a nifedipina, corticosteróides, antibióticos como a tetraciclina, e algumas preparações contendo vitaminas. Comprimidos embalados em blister colorido podem apresentar uma redução de 30 \% a 40 \% no grau de fotodegradação (PIECHOCKI\& THOMA, 2006).

Em um estudo, foi demonstrado o efeito protetor da cor utilizando comprimidos de nifedipina não revestidos (Figura 2).

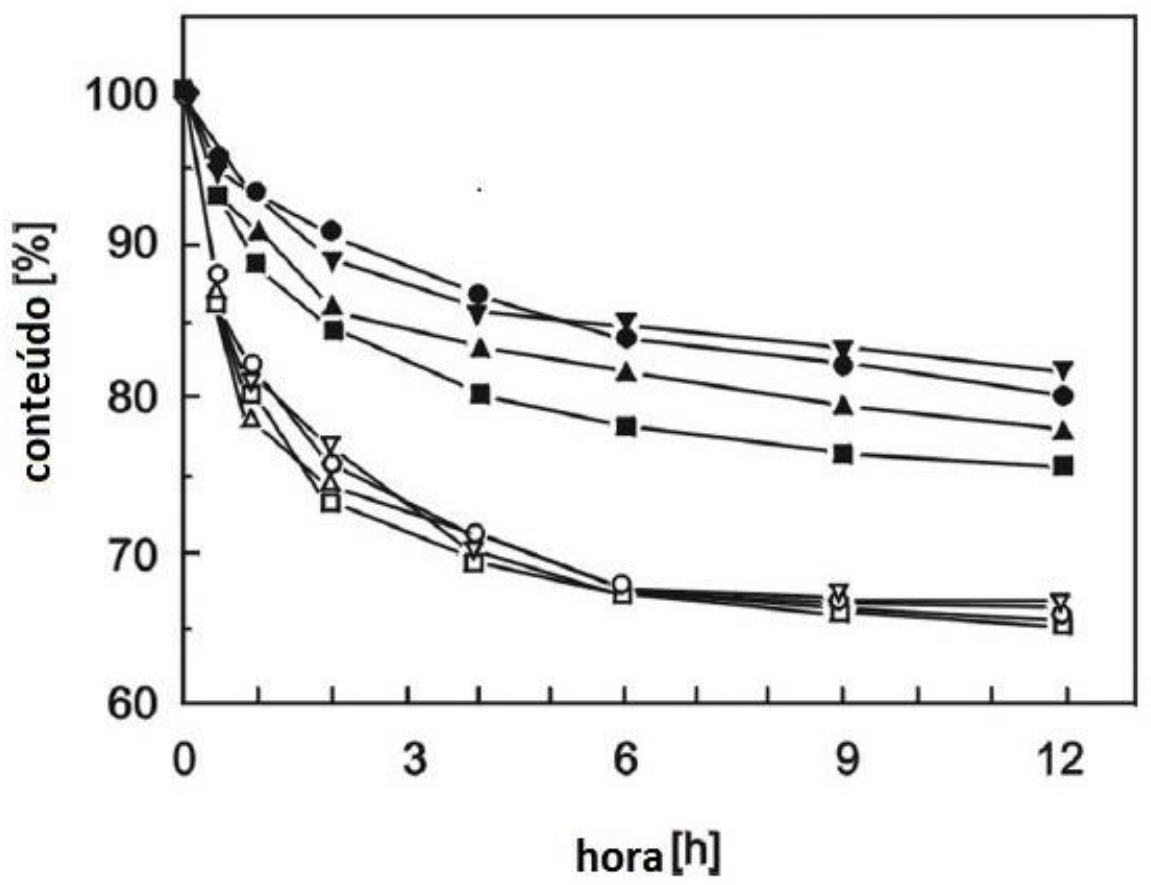

$(\boldsymbol{\nabla})$ blister verde, $(\nabla)$ blíster azul, $(\bullet)$ blíster amarelo, $(O)$ blíster incolor com aditivo de absorção de luz UV, $(\boldsymbol{\Delta})$ blíster vermelho, $(\Delta)$ blíster incolor, $(\mathbf{\square})$ blíster alaranjado, $(\square)$ sem blíster.

Figura 2-Diferenças entre o efeito fotoprotetor de blisters coloridos. Fonte: PIECHOCKI \& THOMA, 2006

O blíster com alvéolos na cor verde apresentou maior efeito fotoprotetor. Após nove horas de irradiação, houve uma perda de menos de $15 \%$ do conteúdo dos comprimidos. 
Também foram encontrados efeitos fotoprotetores no blister com alveolos vermelho, alaranjado e amarelo. O blíster com alvéolos azuis e os incolores tiveram pouco efeito fotoprotetor nos comprimidos de nifedipina. Após seis horas de irradiação, houve uma perda de mais de $30 \%$ do conteúdo. Portanto, é evidente que a utilização de um blíster com a cor adequada pode ser um fotoprotetor eficiente (PIECHOCKI\& THOMA, 2006).

\section{CONCLUSÃO}

O filmede PVC é um material transparente, de baixocusto, e oferece uma estabilidade adequada para um grande número de produtos farmacêuticos. Contudo, estes filmes oferecem pouca proteção contra a umidade, além de impactos ambientais.Para proteção contra a umidade e oxigênio, encontram-se os materiais com maior função de barreira como laminados combinados de PVC/PVDC, PVC/COC/PVC eAclar®. Filmes do tipo Alumino/Alumínio oferecem proteção máxima contra a luz, umidade e oxigênio.

A escolha de uma embalagem adequada para os fármacos é fundamental para que não ocorram alterações na composição do produto, garantindo a estabilidade e proteção até que a última dose seja administrada. A escolha de uma determinada cor do blister para atender às especificações requeridas pela legislação brasileira e a sua adequação para medicamentos fotossensíveis deve ser criticamente avaliada em estudos de estabilidade, dado que de acordo com alguns autores pode-se haver variação na proteção a fotodegradação.

A instabilidade de um medicamento pode levar a sua inefetividade e, consequentemente, gerar danos à saúde e segurança do paciente.

\section{REFERÊNCIAS}

ALVES, R. M. V. et al. Embalagens plásticas e de vidro para produtos farmacêuticos: avaliação das propriedades de barreira à luz. Rev. Ciên. Farm. Basica Apl., v. 29, n. 2, p. 169-180, 2008.

AMARAL, T.R.; RODRIGUES, L. B. O.; CASTRO, G. A.Padronização dos desenhos utilizados nos processos de blistagem. Pós em Revista,v. 5,p. 299-311, 2012. 
BERASAY, S. Blísters para fármacos: Segurança e proteção. Revista Pharmceutical Technology, v.4, n.4, p. 58 - 59, 2005.

BRASIL. Ministério da Saúde. Agência Nacional de Vigilância Sanitária. Resolução - RDC n. 1, de 29 de julho de 2005. Autoriza a publicação do Guia para a Realização de Estudos de Estabilidade. Brasília: Diário Oficial da União, 2005. Disponível em <http://www.anvisa.gov.br/medicamentos/legis/01_05_re_comentada.pdf>

CARREIRA, M.S.da C. Desenvolvimento de uma ferramenta para o acondicionamento de medicamentos na industria farmacêutica. 117f. Dissertação de Mestrado. Universidade de Coimbra. 2009.

CRONIMO GROUP. Blister process in pharmaceuticalindustry. Disponível em: $<$ http://www.technocom.at/download/leaflet/BlisterPresentation.pdf $\geq$

FORCíNIO, H. "Criando alternativas de embalagem", PharmaceuticalTechnology,v.4, n.5, p.28-44,2002.

GARCIA, S. Para cada tipo de cápsula ou comprimido há um blíster específico que deve ser produzido, 2001. Disponível em: <http://www.cellopress.com.br/sueligarcia/web/imprensa /imprensa_006.htm>.Acesso em 29 de junho de 2016.

HOLLANDER, R. Substituição dos filmes de PVC e Laminados de Alumínio. Pharmaceutical Technology., v.2, n.1, p.22-31, fev.1998.

HUNTE, N. Blister packaging materials. Pharmaceutical \& Medical Packaging News. n.7, p.36-38, 1999.

JNANADEVA, B.M. Role of packaging material on Pharmaceutical product stability. Disponível em:<http://www.ipapharma.org/events/stability/jbhat.pdf>. Acesso em junho de 2016.

JORGE, N. Embalagens para alimentos. São Paulo: Cultura Acadêmica: Universidade Estadual Paulista, Pró-Reitoria de Graduação, 2013. 194 p 
LIMA, B.R.de. A importância do desenvolvimento da embalagem na indústria farmacêutica.Trabalho de Conclusão de Curso. Faculdade de Tecnologia da Zona Leste, São Paulo, 2010.

LUSINA, M.; CONDRIC, T.; JADRANKA, T.; MARIJANA, P.; POZAIC, L.; MUSULIN, N. Stability study of losartan/hydrochlorothiazide tablets. International Journal of Pharmaceutical., v.291, p.127-137, 2005.

NASCIMENTO, A. Blistagem é alternativa para acondicionar medicamentos. Revista Controle de Contaminação, n.90, ano 09, p. 14-19, 2006.

NICOLOSI, M. Processo de embalagem exige entrosamento entre indústria e fornecedores. Revista Controle de Contaminação, n.108, ano 10, p. 14-19, 2008.

NÓBREGA, I.M.F.; GRANGEIRO, J.S.; Silva,R.M.F.; Rolim, N.P.J.; Albuquerque MM. Estudo de estabilidade de comprimidos de Captopril $25 \mathrm{mg}$ acondicionados em blister frente a diferentes tipos de filmes moldáveis. Rev Bras Farm, 87(4):128-31, 2006.

PIECHOCKI, J.T., THOMA, K. Pharmaceutical Photostability and Stabilization Technology. CRCPress, 2006. PILCHIK, R. Pharmaceutical Blister Packaging, Part I: rationale and Materials. Pharmaceutical Technology, v.24, n.11, p.68-77, 2000.

QUINTAS, T.T. Re-design do medicamento allestra 20. 2013. 40 f. Trabalho de Conclusão de Curso (Especialização) - Universidade Tecnológica Federal do Paraná, Curitiba, 2013.

REMINGTON, A.G. A Ciência e a Prática da Farmácia. $20^{\circ}$ ed., Rio deJaneiro: Editorial Guanabara Koogan S.A., 2004. 2208p.

RODRIGUES, L.N.C.; FERRAZ, H.G. Embalagem farmacêutica tipo blíster: escolha de um filme adequado para fármacos sensíveis à umidade. Revista Analytica, n.28, p. 80-86, 2007.

STINGHEL, M.L. Avaliação de embalagens primárias para comprimidos de Cloridrato de Metformina $850 \mathrm{mg}$ através de estudos de estabilidade. [TCC - Especialização]. Rio de Janeiro: Instituto de Tecnologia em Fármacos/Farmanguinhos, Fundação Oswaldo Cruz, 2014. 